\title{
GABAergic neuronal development in the embryonic mesencephalon of mice
}

\author{
Mun-Ki Kim ${ }^{1}$, Si-Joon Lee ${ }^{1}$, Anju Vasudevan ${ }^{2}$, Chung-Kil Won ${ }^{1 *}$ \\ ${ }^{1}$ Institute of Animal Medicine \& Department of Veterinary Medicine, Gyeongsang National University, Jinju 52828, Korea \\ ${ }^{2}$ Angiogenesis and Brain Development Laboratory, McLean Hospital/Harvard Medical School, Belmont, MA 02478, USA
}

\begin{abstract}
This study presents neurogenesis and neuronal migration patterns of gamma-aminobutyric acid-ergic (GABAergic) neurons during mesencephalic development of mouse. After neurons from embryonic day (E) 10-16 were labelled by a single injection of 5-bromo-2'-deoxyuridine (BrdU), immunohistochemistry was performed. Neurogenesis were mainly generated in the mesencephalic region at E10 to E13. After E14, BrdU positive cells were observed only in the dorsal mesencephalon. GABAergic neurons were mainly originated in the ventrolateral region of the mesencephalon at the early embryonic stage, especially at E11 to E13. E10-labeled cells showed positive for GABAergic neuron in the basal plate of the mesencephalon at E13. At E15, GABAergic neurons were observed in the entire basal plate and some regions of the ventral and dorsal mesencephalon. They were present in the whole basal plate, the ventral and dorsal mesencephalon of E17, spreading more outward of the mesencephalon at P0. Our study demonstrates that major neurogenesis of GABAergic neurons occurs at E11 to E13. However, neuronal migration continues until neonatal period during mesencephalic development.
\end{abstract}

Keywords: development, GABA, mesencephalon, neurogenesis, neuronal migration

*Corresponding author

Chung-Kil Won

Department of Veterinary Medicine, Gyeongsang National University, 501 Jinju-daero, Jinju 52828, Korea

Tel: $+82-55-772-2351$

Fax: +82-55-772-2349

E-mail: wonck@gnu.ac.kr

ORCID

Chung-Kil Won

https://orcid.org/0000-0002-3105-1869

Mun-Ki Kim

https://orcid.org/0000-0002-7397-2236

Conflict of Interest

The authors declare no conflicts of interest.

Received: October 30, 2019

Revised: November 13, 2019

Accepted: November 15, 2019

\section{Introduction}

Neurogenesis and neuronal migration are essential development steps in the midbrain and are maximally active in the embryo [1-3]. Neurogenesis gradients offer clues as to where in the neuroepithelium the germinal site may be located and what routes migrating cells take to reach their final destination [4].

Gamma-aminobutyric acid (GABA) neurons are key players in the mammalian central nervous system (CNS) [5-7]. Their impairment is involved in the etiology of several neurological disorders [8]. GABAergic neurons take in various forms and distribution in every region of the CNS. It has been demonstrated that the pattern of development of GABAergic neurons is distinct from that of other types of neurons in the cerebral cortex. Most cortical neurons are generated in the ventricular zone and then migrate radially along the radial glial fibers to each layer [9]. In contrast, GABAergic neurons are produced in the ventricular zone and ganglionic eminence of the forebrain and are distributed to the cortex by tangential migration [10-12]. It is interesting to clarify whether these developmental patterns for GABAergic neurons are common in other layered structures. Thus, GABAergic neurogenesis and neuronal migration are also important key development process in the CNS [13-16].

However, neurogenesis and neuronal migration of GABAergic neurons in the development of mesencephalon have not been well understood yet. Therefore, the objective of the present study was to demonstrate early and later neurogenesis and neuronal migration of GABAergic neurons in the mesencephalon of mouse.

\section{Materials and Methods}

\section{Animals}

Timed pregnant CD1 mice were purchased from Charles River Laboratories (USA). Colonies of GAD65-green fluorescent protein (GFP) mice were maintained in an institutional animal facility. A female mouse housed with a male mouse for 15-17 hours was examined for the presence of vaginal plugs 
at 9:00 A.M. The presence of the plug indicated conception. The day of plug discovery was designated embryonic day 0 (E0). The embryos were removed by deep inhalation anesthesia by the mother of mouse with isoflurane, and three were used in each group regardless of male and female. Animal experiments were in full compliance with the NIH Guide for the Care and Use of Laboratory Animals. They were approved by the McLean Institutional Animal Care and Use Committee.

\section{5-Bromo-2'-deoxyuridine (BrdU) labeling and immu- nohistochemistry}

To understand neurogenesis, a single 5-bromodeoxyuridine $(50 \mu \mathrm{g} / \mathrm{g}$ body weight, i.p; Sigma) injection was administrated to E10, E11, E12, E13, E14, and E16 pregnant dams and each embryo was removed after $24 \mathrm{~h}$. For neuronal migration study, BrdU labeling was achieved by administering a single BrdU injection (50 $\mu \mathrm{g} / \mathrm{g}$ body weight, i.p; Sigma) to pregnant dams carrying E10 mice. Embryos were removed at E13, E15, E17 and postnatal day 0 (P0) and decapitated. Embryonic brains were immersed in zinc fixative (BD Pharmingen, USA) for $24 \mathrm{~h}$ and then processed for paraffin wax histology. BrdU immunohistochemistry was performed for $10 \mu \mathrm{m}$ thick paraffin embedded sections with a mouse monoclonal anti-BrdU antibody $(1: 75,347580$, BD Pharmingen). Double labeling immunohistochemistry for BrdU and glutamic acid decarboxylase (GAD) 65/GAD67 was performed using rabbit polyclonal anti-GAD65/GAD67 (1:500, AB1511, Millipore, USA). Double labeling immunohistochemistry for BrdU and GAD was performed using sheep polyclonal anti- GAD65/GAD67 (1:200, AB1542, Millipore). Streptavidin Alexa fluor 488 conjugate and Streptavidin Alexa fluor 594 conjugate (Molecular Probes, USA) were used as secondary antibodies.

\section{Results}

\section{Neurogenesis in the mesencephalon}

$\mathrm{BrdU}$ is integrated into the DNA of S-phase progenitor cells. It serves as a stable marker for cells born around the time of injection. Thus, BrdU has been widely used to understand neurogenesis and neuronal migration in the developing brain $[17,18]$. To observe mesencephalic neurogenesis, BrdU labelled mouse fetus was collected and immunohistochemistry was performed. Many E10 to E12-originated BrdU positive cells were observed to be uniformly distributed in the entire mesencephalic neuroepithelium region (Fig. 1A-C). E14-originated BrdU positive cells were only observed in the dorsal mesencephalon region (Fig. 1D). A few E16-originated BrdU positive cells were detected in the mesencephalon (Fig. 1E).

\section{GABAergic neurogenesis and neuronal migration}

To investigate when and where GABAergic neurons were originated in the mesencephalon, BrdU was pulsed in CD1
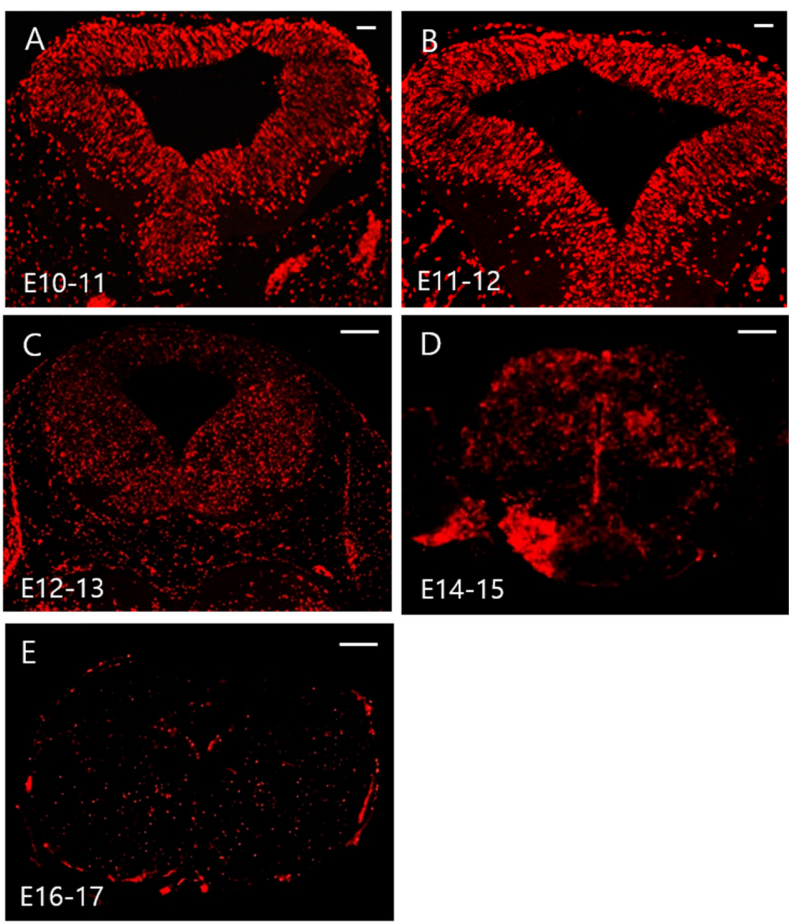

Fig. 1. Neurogenesis profiles in the mesencephalon. A single 5bromo-2'-deoxyuridine (BrdU) was injected at E10 to E16 and each sample was removed after $24 \mathrm{~h}$. E10-E12 originated BrdU positive cells were observed to be uniformly distributed in the mesencephalic region (A-C). E14-originated BrdU positive cells were only observed in the dorsal mesencephalon (D). A few E16-originated BrdU positive cells were detected in the mesencephalon (E). Scale bars $=50 \mu \mathrm{m}(\mathrm{A}, \mathrm{B}) ; 100 \mu \mathrm{m}(\mathrm{C}-\mathrm{E})$.

mice at E11, E12, E13, and E14 and each sample was collected after $24 \mathrm{~h}$. In BrdU labeled cells at E11, E12, and E13, GAD positive cells were observed in the ventrolateral region (Fig. 2B-E). A higher magnification of the ventrolateral region revealed GAD/BrdU double positive cells (Fig. 2G). For cells labeled with BrdU after E14, a connection between GAD and BrdU positive cells was no longer observed in the mesencephalon (Fig. 2F).

In the neuronal migration study, a single pulse BrdU was administrated at E10. Migration of GAD and BrdU positive cells were followed at E13, E15, E17, and P0 (Fig. 3). GAD expression was observed in the basal plate region at E13 (Fig. 3A). At E15, GAD expression was observed in the entire basal plate and some regions of the ventral and dorsal mesencephalon (Fig. 3B). Clear expression of GAD was observed in the entire mesencephalon of E17, spreading more outward of the mesencephalon at P0 (Fig. 3C-D). We used a GFP knock-in mouse in which a GFP gene was introduced into the gene for GAD 65 and GABAergic neurons were fluorescent. BrdU positive cells were shown to migrate through dorsal and ventral routes while GFP/BrdU double positive cells were observed at E17 in the ventrolateral mesencephalon (Fig. 4D). 

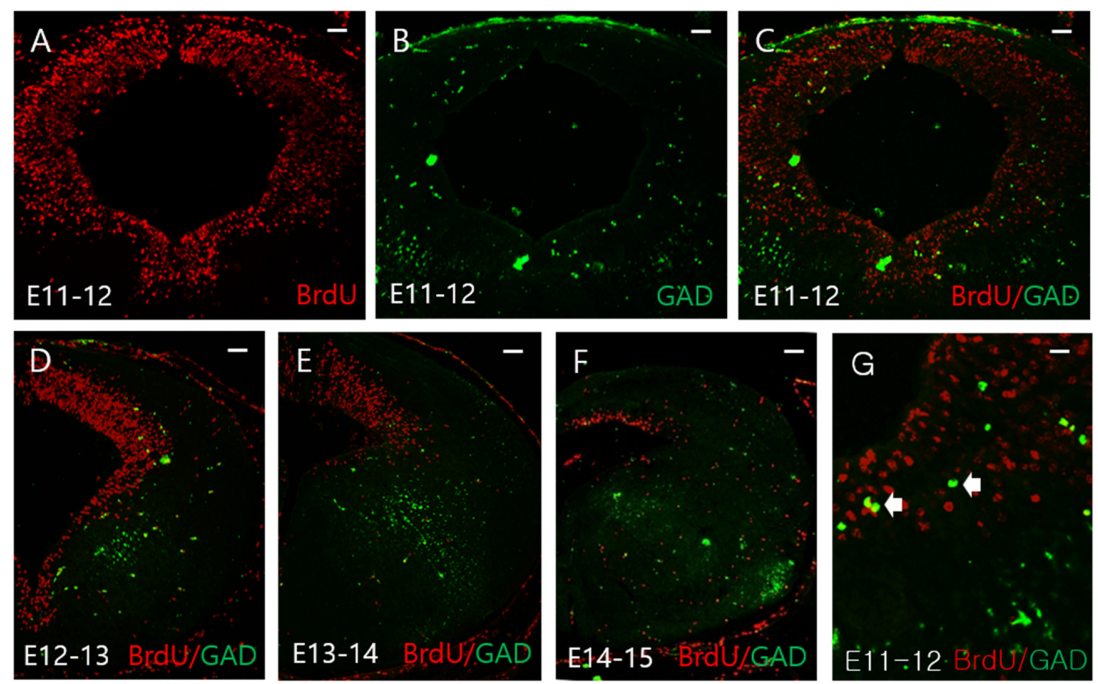

Fig. 2. Glutamic acid decarboxylase (GAD) expression in the mesencephalon. A single 5-bromo-2'-deoxyuridine (BrdU) was pulsed at E11 to E14 and each sample was collected after $24 \mathrm{~h}$ in CD1 mice. At E11-E13, GAD positive cells were observed in the ventrolateral regions (B-E). Higher magnification of $\mathrm{C}$ showed GAD/BrdU double positive cells (G; white arrows). Many GAD positive cells were seen in E13-labeled cells (E). After E14-labeled cells, GAD positive cells were no longer observed in the mesencephalon. Scale bars $=50 \mu \mathrm{m}(\mathrm{A}-\mathrm{F}) ; 10 \mu \mathrm{m}(\mathrm{G})$.
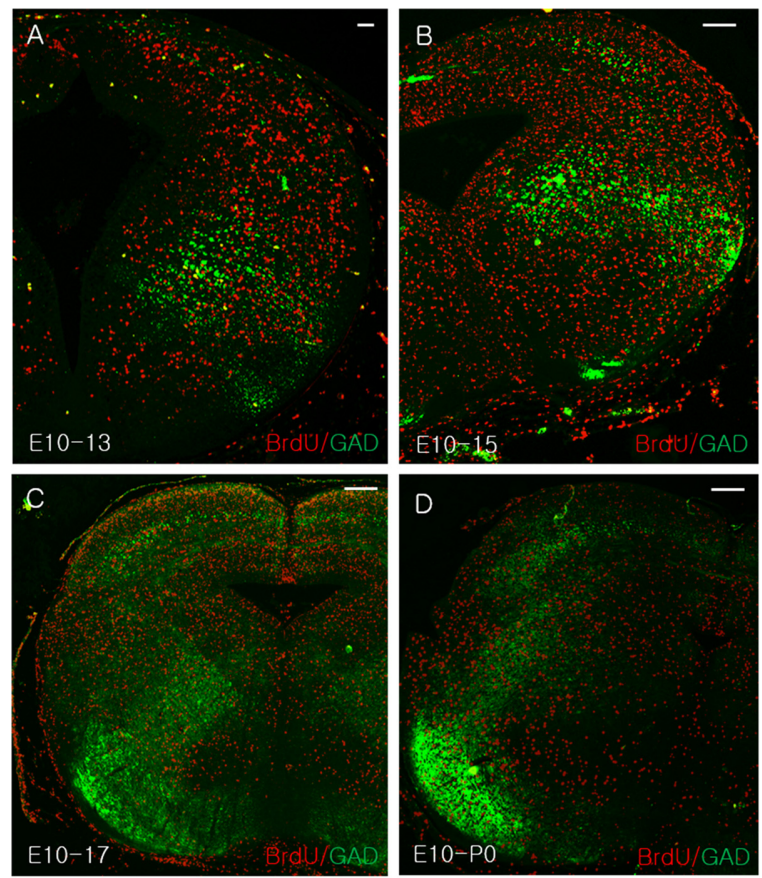

Fig. 3. Glutamic acid decarboxylase (GAD) expression in the developing mesencephalon. A single 5-bromo-2'-deoxyuridine (BrdU) was administrated at E10 and migration of GAD/BrdU positive cells were followed at E13 to P0. E10 labeled BrdU positive cells were observed to be uniformly distributed at E13$\mathrm{P} 0$ in the mesencephalon. GAD expression was observed in the basal plate at E13 (A). At E15, GAD positive cells were observed in the basal plate and ventrolateral mesencephalon (B). At E17, clear GAD expression was observed in the whole mesencephalon $(C)$, spreading more outward of the mesencephalon at P0 (D). Scale bars $=25 \mu \mathrm{m}(\mathrm{A}, \mathrm{B}) ; 50 \mu \mathrm{m}(\mathrm{C}, \mathrm{D})$.
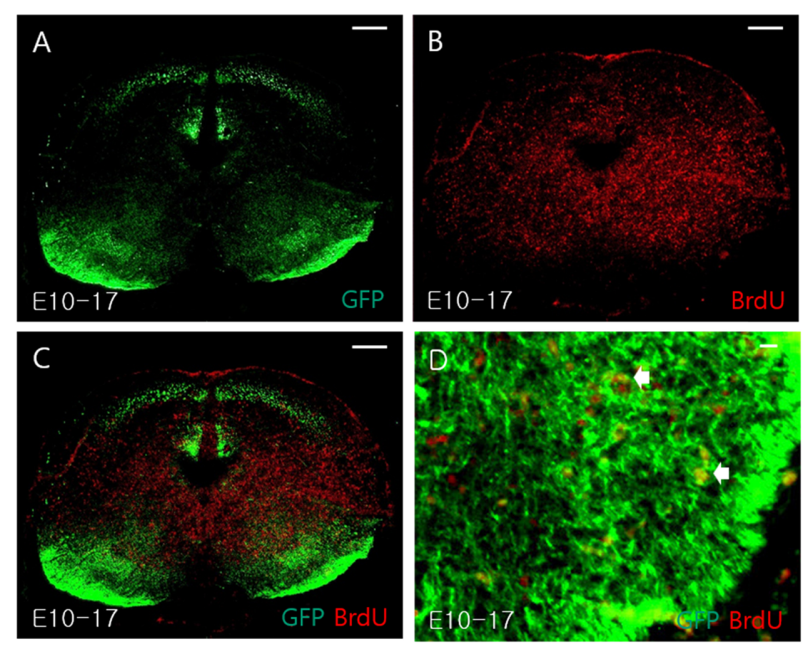

Fig. 4. Glutamic acid decarboxylase (GAD) expression in green fluorescent protein (GFP) knock-in mouse. A single 5-bromo2'-deoxyuridine (BrdU) was pulsed at E10 and migration of GFP/BrdU expression were followed at E17. BrdU positive cells were shown to migrate through dorsal and ventral routes (B). GFP/BrdU double positive cells were observed at E17 in the ventrolateral mesencephalon ( $\mathrm{D}$; white arrows). Scale bars $=$ $100 \mu \mathrm{m}$ (A-C); $10 \mu \mathrm{m}$ (D).

\section{Discussion}

The GABAergic neuron control several aspects of behavior, play important roles in psychiatric diseases, susceptibility to drugs of abuse and are also important targets for several medical treatments for these diseases $[8,19]$. However, the GABAergic neuronal development in the midbrain 
have been neglected until recently. Studies on neurogenesis using thymidine $\left[{ }^{3} \mathrm{H}\right]$ in the mesencephalon of mice have shown that neurogenesis mainly occurs at E10 and E12 [20]. In our previous study, BrdU-positive cells were observed to be uniformly distributed in the whole mesencephalon at E10E13 [21]. Results of the neurogenesis study using BrdU in the present study corroborated with results of a previous study indicating that neurogenesis was generated predominantly at E10 and E12. Concerning DA neurogenesis, our previous experiments showed that DA neurons mainly originated in the ventricular region of the midbrain at early embryonic stages E10 to E11 and that those neurons after E12 were only observed in the ventral mesencephalon [21]. Our results show that GABAergic neurogenesis, unlike dopamine neurogenesis, occurs mainly in the basal plate at E11E13 and rarely occurs after E14.

The population of GABAergic neurons in adult midbrain is known to regulate glutamatergic and dopaminergic neuronal activity [22]. They have projection targets similar to those of dopaminergic neurons [23]. Neuronal migration seems to be an essential phase in the formation of the anatomical architecture of the mesencephalon. Thus, this study hypothesizes that GABAergic neurons show a similar neuronal migration pattern to that of dopaminergic neurons because GABAergic and dopaminergic neurons are closely related to each other [3]. GABAergic and dopaminergic neurons in the mesencephalon are predominantly originated at early embryonic stages [2,3,21]. TH expression was initially detected at E10 and many E10-labeled BrdU positive cells were found to be merged with dopaminergic neurons in the SN and VTA at E17 [3,21]. In this study, E10-labeled cells showed positive for GAD in the basal plate at E13 and GAD expression were observed in the whole basal plate at E15. They were present in the whole basal plate and the ventral mesencephalon with the passage of time. Moreover, many E10-labeled BrdU positive cells were merged with GABAergic neurons in the ventrolateral mesencephalon at E17 in this study. These results suggested that major neurogenesis of GABAergic neurons occurred at E11 to E13 and that neuronal migration continued until neonatal period during mesencephalic development.

\section{Acknowledgements}

This research was supported by Basic Science Research Program through the National Research Foundation of Korea (NRF) funded by the Ministry of Education (2019018414).

\section{References}

1. Hatten ME. New directions in neuronal migration. Science 2002;297:1660-1663.

2. Li S, Joshee S, Vasudevan A. Mesencephalic GABA neuronal development: no more on the other side of oblivion. Biomol Concepts 2014;5:371-382.

3. Vasudevan A, Won C, Li S, Erdélyi F, Szabó G, Kim KS. Dopaminergic neurons modulate GABA neuron migration in the embryonic midbrain. Development 2012;139:3136-3141.

4. Altman J, Bayer SA. Development of the brain stem in the rat. V. Thymidine-radiographic study of the time of origin of neurons in the midbrain tegmentum. J Comp Neurol 1981; 198:677-716.

5. Barbaresi P. Postnatal development of GABA-immunoreactive neurons and terminals in rat periaqueductal gray matter: a light and electron microscopic study. J Comp Neurol 2010; 518:2240-2260.

6. Fahn S, Côté LJ. Regional distribution of gamma-aminobutyric acid (GABA) in brain of the rhesus monkey. J Neurochem 1968;15:209-213.

7. Mize RR. The organization of GABAergic neurons in the mammalian superior colliculus. Prog Brain Res 1992;90:219248.

8. Benes FM, Berretta S. GABAergic interneurons: implications for understanding schizophrenia and bipolar disorder. Neuropsychopharmacology 2001;25:1-27.

9. Rakic P. Mode of cell migration to the superficial layers of fetal monkey neocortex. J Comp Neurol 1972;145:61-83.

10. Anderson SA, Eisenstat DD, Shi L, Rubenstein JL. Interneuron migration from basal forebrain to neocortex: dependence on Dlx genes. Science 1997;278:474-476.

11. Letinic K, Zoncu R, Rakic P. Origin of GABAergic neurons in the human neocortex. Nature 2002;417:645-649.

12. Tsunekawa N, Yanagawa Y, Obata K. Development of GABAergic neurons from the ventricular zone in the superior colliculus of the mouse. Neurosci Res 2005;51:243-251.

13. Corbin JG, Nery S, Fishell G. Telencephalic cells take a tangent: non-radial migration in the mammalian forebrain. Nat Neurosci 2001;4 Suppl:1177-1182.

14. Marín O, Rubenstein JL. A long, remarkable journey: tangential migration in the telencephalon. Nat Rev Neurosci 2001;2: 780-790.

15. Parnavelas JG. The origin and migration of cortical neurones: new vistas. Trends Neurosci 2000;23:126-131.

16. Nadarajah B, Parnavelas JG. Modes of neuronal migration in the developing cerebral cortex. Nat Rev Neurosci 2002;3: 423-432.

17. Lewandowski TA, Ponce RA, Charleston JS, Hong S, Faustman EM. Changes in cell cycle parameters and cell number in the rat midbrain during organogenesis. Brain Res Dev Brain Res 2003;141:117-128.

18. Soriano E, Del Rio JA. Simultaneous immunocytochemical visualization of bromodeoxyuridine and neural tissue antigens. J Histochem Cytochem 1991;39:255-263.

19. Lahti L, Achim K, Partanen J. Molecular regulation of GABAergic neuron differentiation and diversity in the developing midbrain. Acta Physiol (Oxf) 2013;207:616-627.

20. Bayer SA, Wills KV, Triarhou LC, Ghetti B. Time of neuron origin and gradients of neurogenesis in midbrain dopaminergic neurons in the mouse. Exp Brain Res 1995;105:191-199.

21. Kim MK, Lee SJ, Vasudevan A, Won C. Neurogenesis and neuronal migration of dopaminergic neurons during mesencephalon development in mice. J Biomed Transl Res 2018;19: 125-129.

22. Nair-Roberts RG, Chatelain-Badie SD, Benson E, WhiteCooper H, Bolam JP, Ungless MA. Stereological estimates of dopaminergic, GABAergic and glutamatergic neurons in the ventral tegmental area, substantia nigra and retrorubral field 
in the rat. Neuroscience 2008;152:1024-1031.

23. Korotkova TM, Ponomarenko AA, Brown RE, Haas HL.
Functional diversity of ventral midbrain dopamine and GABAergic neurons. Mol Neurobiol 2004;29:243-259. 